\title{
Multicolor infrared detection realized with two distinct superlattices separated by a blocking barrier
}

\author{
C. C. Chen, H. C. Chen, and C. H. Kuan ${ }^{\text {a) }}$ \\ Department of Electrical Engineering and Graduate Institute of Electronics Engineering, \\ National Taiwan University, Taipei, Taiwan, Republic of China 10617 \\ S. D. Lin and C. P. Lee \\ Department of Electronic Engineering, National Chiao Tung University, Hsinchu, \\ Taiwan, Republic of China 30050
}

(Received 26 November 2001; accepted for publication 4 February 2002)

\begin{abstract}
A multicolor infrared photodetector was realized with two superlattices separated by a blocking barrier. The photoresponse is switchable between $7.5-12$ and $6-8.5 \mu \mathrm{m}$ by the bias polarity, and is also tunable by the bias magnitude in each wavelength regime. In addition, our detector exhibits advantages including little temperature dependence of the spectral response and the same order of responsivity in the two wavelength regimes. The measured peak responsivities in the two regimes are $117 \mathrm{~mA} / \mathrm{W}$ at $9.8 \mu \mathrm{m}$ under $1 \mathrm{~V}$ and $129 \mathrm{~mA} / \mathrm{V}$ at $7.4 \mu \mathrm{m}$ under $-0.8 \mathrm{~V}$, respectively. Also, the detectivities are comparable with the conventional multistack detector. The zero background peak detectivities are $2.3 \times 10^{10} \mathrm{~cm} \mathrm{~Hz}^{0.5} / \mathrm{W}$ at $50 \mathrm{~K}$ and $9.8 \mu \mathrm{m}$ under $0.7 \mathrm{~V}$, and 8.7 $\times 10^{10} \mathrm{~cm} \mathrm{~Hz}^{0.5} / \mathrm{W}$ at $70 \mathrm{~K}$ and $7.4 \mu \mathrm{m}$ under $-0.7 \mathrm{~V}$. (C) 2002 American Institute of Physics.
\end{abstract}

[DOI: $10.1063 / 1.1466537$ ]

An infrared photodetector capable of multicolor operation is essential in various applications such as aerospace observation, target discrimination, and temperature sensing. Because of the flexibility of the band engineering, structures utilizing the quantum well ${ }^{1-5}(\mathrm{QW})$ and superlattice ${ }^{6,7}(\mathrm{SL})$ were reported to realize the multicolor detector. In this letter, we adopt two distinct SLs separated by a blocking barrier to achieve multicolor detection. The photoresponse of our photodetector is switchable between two wavelength regimes $(6-8.5$ and $7.5-12 \mu \mathrm{m})$ by the bias polarity, and is also tunable by the bias magnitude in each wavelength regime. Compared with the conventional multistack detector, our detector shows the advantages of little temperature dependence of the spectral responsivity. This is particularly difficult to be achieved by conventional multistack QW structures.

The advantages of our detector are due to the different operational mechanisms from conventional multistack detectors. To show these, the operational mechanisms of conventional detectors are introduced. In a multistack quantum-well infrared photodetector (QWIP), the various stacks are separated either by heavily doped conducting layers or thick barriers. In the former case, the fraction of the differential resistance determines the individual contribution to the photoresponse from each stack. ${ }^{8}$ The stack with higher differential resistance contributes more to the photoresponse. In general, the short-wavelength stack has higher differential resistance than the long-wavelength one at a low applied voltage. Therefore, the long-wavelength spectral responsivity can be observed only under a high bias when the shortwavelength stack is driven into saturation and has comparable or lower differential resistance. Since the differential resistance of each stack is determined primarily by the voltage characteristic of the dark current, it is difficult to control

${ }^{a)}$ Electronic mail: kuan@cc.ee.ntu.edu.tw the responsivity in the design phase. Because up to now, no theoretical model can accurately predict the voltage characteristic of the dark current of each detection stack over a wide range of applied biases. Besides, the dark current is also susceptible to the variation of the operational temperature. Therefore, the photoresponse in such detectors may change with the operational temperature.

The other type of conventional multistack detector utilizes a thick barrier to separate the detection stacks. ${ }^{9-11} \mathrm{Un}$ der an external bias, domains with different electric fields occur in such detectors as the case of a single-color QWIP. Generally, high-field domain first forms in the shortwavelength stack under a low applied bias, and the photoresponse is dominated by the short wavelength. Longwavelength photoresponse can only be observed under highbias condition along with the accompaniment of the shortwavelength one. Both types of multistack photodetectors and several other QW-based structures ${ }^{3,12}$ for multicolor detection also suffer those disadvantages.

To overcome the disadvantages, the SLs were adopted as the detection stacks of the multicolor detector. Unlike QWs, the electron wave function in the SL well overlaps the ones in the neighboring wells. The coupling of the wave functions results in the formation of the miniband. Since the electrons in the miniband can tunnel through the entire SL, the SL alone is a low-resistance structure. It is the low-resistance characteristic that avoids the aforementioned disadvantages. In the following, we present the design and performance of our multicolor infrared photodetector with a separating barrier sandwiched between two distinct SLs.

The inset of Fig. 1 shows the structure of our photodetector under a positive bias. The voltage polarity is taken as positive if a high potential is applied on the top contact. The structure contains sequentially a $500 \mathrm{~nm}$ bottom contact layer, a 14-period bottom SL, a blocking barrier, another 


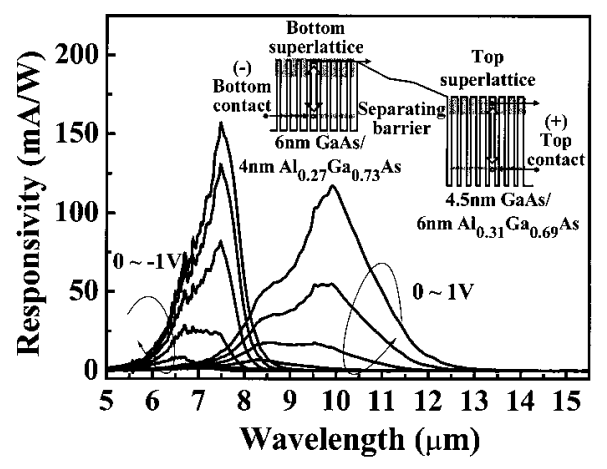

FIG. 1. Measured spectral responsivity at $30 \mathrm{~K}$ under several bias voltages. The inset shows the structure of the photodetector under a positive bias.

14-period top SL, and a $400 \mathrm{~nm}$ top contact layer. Each period of the bottom and top SLs is, respectively, composed of a $6 \mathrm{~nm} \mathrm{GaAs}$ well and $4 \mathrm{~nm} \mathrm{Al}_{0.27} \mathrm{Ga}_{0.73} \mathrm{As}$ barrier, and a $4.5 \mathrm{~nm} \mathrm{GaAs}$ well and $6 \mathrm{~nm} \mathrm{Al}_{0.31} \mathrm{Ga}_{0.69} \mathrm{As}$ barrier. The blocking barrier consists of a $60 \mathrm{~nm} \mathrm{Al} \mathrm{Al}_{0.27} \mathrm{Ga}_{0.73} \mathrm{As}$ layer, a $50 \mathrm{~nm}$ graded $\mathrm{Al}_{x} \mathrm{Ga}_{1-x}$ As layer with $x$ increasing from 0.27 to 0.31 , and a $60 \mathrm{~nm} \mathrm{Al}_{0.31} \mathrm{Ga}_{0.69}$ As layer. Both the contact layers and the SL wells are doped with $1 \times 10^{18} \mathrm{~cm}^{-3}$ of Si. The blocking barrier and the SL barriers are left undoped.

The operational mechanisms are also shown in the inset of Fig. 1. Because of the low-resistance characteristic of the SL, the applied voltage is almost totally dropped on the separating barrier. The photoelectrons in the second miniband of the bottom SL can tunnel through the separating barrier due to the electric field applied on the barrier. The escaped photoelectrons result in net positive charges to attract electrons from the bottom contact and cause the photocurrent in the external circuit. On the contrary, the escaped photoelectrons in the top SL cause electrons from the top contact and result in internal current circulation, as shown in the inset of Fig. 1. Therefore, only the bottom SL is active under a positive bias. In the same way, only the top SL is active under a negative bias. This characteristic makes the spectral responsivity switchable by the bias polarity between the two wavelength regimes corresponding to the respective miniband transitions of the top and bottom SL. Particularly, the photoelectrons with different energies relative to the edge of the separating barrier exhibit different voltage dependences of the tunneling probability. As a result, the spectral responsivity is tunable by the magnitude of the applied voltage. For the detailed mechanism, please refer to our previous work. ${ }^{7}$ In brief, under a low bias magnitude, the photoelectrons generated by the short-wavelength radiation have higher energy and tunneling probability to contribute to the photoresponse. The spectral responsivity is primarily in the short-wavelength part at the low bias magnitude under both voltage polarities. However, under a high bias magnitude, the tunneling probability of the photoelectrons in the bottom state of the second miniband increases, and the long wavelength can dominate the spectral responsivity. Therefore, the blocking layer not only isolates the photoelectrons from the top and bottom SLs but also serves as a high-pass energy filter for the photoelectrons. Since the SLs have no voltage drop at operation, the spectral responsivity in each detection stack is not affected by the differential resistance as the conventional multistack QWIP. In addition, the responsivity can be designed to be

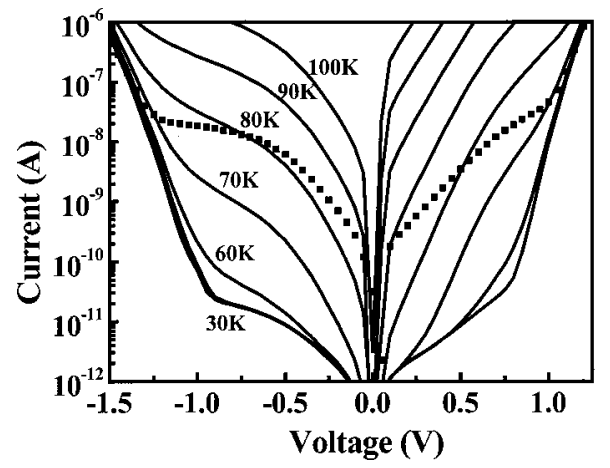

FIG. 2. Dark current (solid curves) at temperatures ranging from 30 to 100 $\mathrm{K}$ and background photocurrent (solid squares) at $20 \mathrm{~K}$.

comparable under both bias polarities by adjusting the barrier height. Next, we will show the measured characteristics of our photodetector. The experimental results agree with our design principles.

The measured spectral responsivity shows little temperature dependence and the representative one at $30 \mathrm{~K}$ is shown in Fig. 1. Under the positive bias, the spectral responsivity is dominated by the bottom SL, and is tunable in 7.5-12 $\mu \mathrm{m}$ through the magnitude of the applied bias. While under the negative bias, the spectral responsivity is dominated by the top SL, and is also tunable in $6-8.5 \mu \mathrm{m}$. It is noted that the peak and shoulder positions under various magnitudes of the applied bias remain the same without the Stark effect occurring at both bias polarities. This indicates the voltage drop on the SL is negligible. Also noted in Fig. 1 is the same order of responsivity at both voltage polarities, which is difficult to achieve by using the conventional multicolor QW structure. In addition, the detector is insensitive to the operational temperature. To show this, the integral difference of the spectral responsivity between two temperatures under specific applied voltage is defined as

$$
\epsilon\left(T_{1}, T_{2}, V\right)=\frac{\int\left|R\left(\lambda, T_{1}, V\right)-R\left(\lambda, T_{2}, V\right)\right| d \lambda}{\int R\left(\lambda, T_{1}, V\right) d \lambda},
$$

where $R(\lambda, T, V)$ is the spectral responsivity at temperature $T$ and voltage $V$. The integral difference of the spectral responsivity of our detector is less than $3 \%$ with temperature ranging from 20 to $70 \mathrm{~K}$.

In addition to isolating the top and bottom SL, the separating barrier also reduces the dark current by preventing the conduction from the first-miniband electrons. In Fig. 2, dark current at various temperatures is shown as the solid curves and the background photocurrent at $20 \mathrm{~K}$ is represented by the solid squares. Comparing the solid curves and squares, the background-limited performance temperature is below 60 $\mathrm{K}$ under $0.5 \mathrm{~V}$, and $80 \mathrm{~K}$ under $-0.75 \mathrm{~V}$.

In order to evaluate the detectivity, the noise performance was measured from 1 to $8 \mathrm{kHz}$, which is limited by the noise and bandwidth of our amplifier. The current noise power spectral density (PSD) at $77 \mathrm{~K}$ was measured with our detector immersed in a $77 \mathrm{~K}$ liquid-nitrogen dewar under careful electrical and optical isolation. With sophisticated calibration of the system noise, the detector noise under the positive bias was extracted from the total measured noise, ${ }^{13}$ 


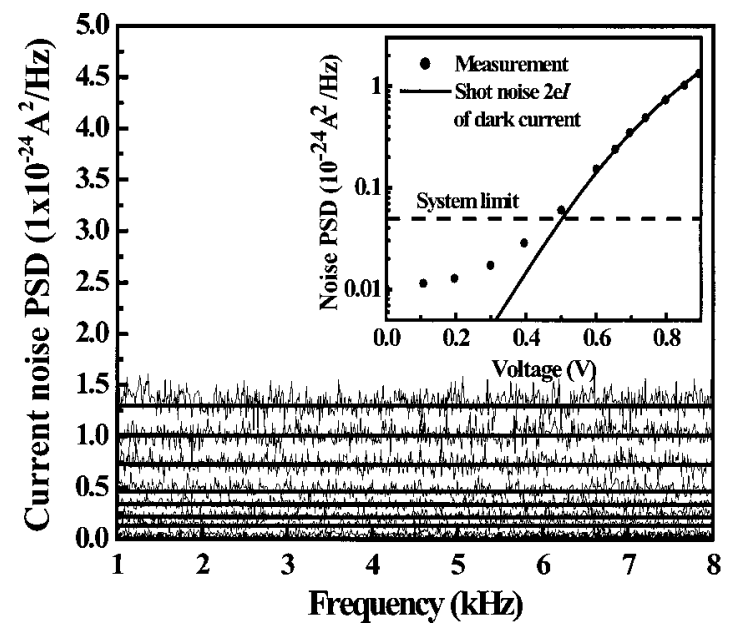

FIG. 3. Measured current noise PSD with the sample immersed in $77 \mathrm{~K}$ liquid nitrogen. Each line corresponds to each point in the inset. The solid curve represents the estimated shot noise calculated with dark current at 77 $\mathrm{K}$. The dashed line indicates the minimum resolvable noise of our measurement system.

and is shown in Fig. 3. The noise of our detector is white noise in the frequency range of our measurement system. Also shown in the inset are the measured noise data and the shot noise $2 e I_{d}$ calculated with the dark current at $77 \mathrm{~K}$. The dashed line in the inset represents the estimated minimum resolvable noise of our noise measurement system. It is observed that the measured noise PSD agrees with the estimated shot noise at $77 \mathrm{~K}$ for voltage larger than $0.5 \mathrm{~V}$. Therefore, it is concluded that the noise source comes from the shot noise of the electrons tunneling trough the blocking barrier. Since the dark current at $77 \mathrm{~K}$ under the negative bias is much smaller than that under positive bias, it is not resolvable in our noise measurement system. In the following evaluation of detectivity, we assume the noise is also the shot noise of the dark current.

The zero background peak detectivity ${ }^{14}$ calculated with the shot noise of our detector is $2.3 \times 10^{10}(1.3$ $\left.\times 10^{11}\right) \mathrm{cm} \mathrm{Hz}^{0.5} / \mathrm{W}$ at $50 \mathrm{~K}(40 \mathrm{~K})$ and $9.8 \mu \mathrm{m}$ under $0.7 \mathrm{~V}$, and is $8.7 \times 10^{10}\left(5.7 \times 10^{11}\right) \mathrm{cm} \mathrm{Hz}^{0.5} / \mathrm{W}$ at $70 \mathrm{~K}(40 \mathrm{~K})$ and $7.4 \mu \mathrm{m}$ under $-0.7 \mathrm{~V}$. Compared with the conventional mul- tistack QWIP, which achieved responsivity of $0.75 \mathrm{~A} / \mathrm{W}$ and detectivity of $4 \times 10^{11} \mathrm{~cm} \mathrm{~Hz}^{0.5} / \mathrm{W}$ at $40 \mathrm{~K}$ and $8.8 \mu \mathrm{m},{ }^{1}$ our detector shows lower responsivity but comparable detectivity.

In summary, we have designed and fabricated an infrared photodetector with two SLs separated by a blocking barrier. Our detector is capable of multicolor operation. The spectral responsivity of our photodetector is switchable by the bias polarities and is tunable by the bias magnitude. In addition, our detector responsivity is insusceptible to the variation of the operational temperature. These show the structure is very appropriate to realize multicolor infrared photodetectors.

This project is supported by the National Science Council under Contract No. NSC 91-2215-E-002-007.

${ }^{1}$ I. Gravé, A. Shakouri, N. Kuze, and A. Yariv, Appl. Phys. Lett. 60, 2362 (1992).

${ }^{2}$ K. L. Tsai, K. H. Chang, C. P. Lee, K. F. Huang, J. S. Tsang, and H. R. Chen, Appl. Phys. Lett. 62, 3504 (1993).

${ }^{3}$ A. Köck, E. Gornik, G. Abstreiter, G. Böhm, M. Walther, and G. Weimann, Appl. Phys. Lett. 60, 2011 (1992).

${ }^{4}$ E. Martinet, E. Rosencher, F. Luc, Ph. Bois, E. Costard, and S. Delaitre, Appl. Phys. Lett. 61, 246 (1992).

${ }^{5}$ H. C. Liu, J. Li, J. R. Thompson, Z. R. Wasilewski, M. Buchanan, and J. G. Simmons, IEEE Electron Device Lett. 14, 566 (1993).

${ }^{6}$ M. C. Hsu, C. H. Kuan, and S. Y. Wang, Appl. Phys. Lett. 77, 2240 (2000).

${ }^{7}$ C. C. Chen, H. C. Chen, M. C. Hsu, W. H. Hsieh, and C. H. Kuan, Appl. Phys. Lett. 91, 943 (2002).

${ }^{8}$ L. C. Lenchyshyn, H. C. Liu, M. Buchanan, and Z. R. Wasilewski, J. Appl. Phys. 79, 8091 (1996).

${ }^{9}$ A. Shakouri, I. Gravé, Y. Xu, and A. Yariv, Quantum Well Intersubband Transition Physics and Devices, edited by H. C. Liu, B. F. Levine, and J. Y. Anderson (Kluwer Academic, Dordrecht, 1994), p. 291.

${ }^{10}$ M. Z. Tidrow, X. Jiang, S. S. Li, and K. Bacher, Appl. Phys. Lett. 74, 1335 (1999).

${ }^{11}$ Y. Zhang, D. S. Jiang, J. B. Xia, L. Q. Cui, C. Y. Song, Z. Q. Zhou, and W. K. Ge, Appl. Phys. Lett. 68, 2114 (1996).

${ }^{12}$ K. Kheng, M. Ramsteiner, H. Schneider, J. D. Ralston, F. Fuchs, and P. Koidl, Appl. Phys. Lett. 61, 666 (1992).

${ }^{13}$ C.-Y. Chen and C.-H. Kuan, IEEE Trans. Instrum. Meas. 77, 49 (2000).

${ }^{14}$ S. D. Gunapala, S. V. Bandara, A. Singh, J. K. Liu, S. B. Rafol, E. M. Luong, J. M. Mumolo, N. Q. Tran, D. Z.-Y. Ting, J. D. Vincent, C. A. Shott, J. Long, and P. D. LeVan, IEEE Trans. Electron Devices 47, 963 (2000). 
Applied Physics Letters is copyrighted by the American Institute of Physics (AIP). Redistribution of journal material is subject to the AIP online journal license and/or AIP copyright. For more information, see http:/ojps.aip.org/aplo/aplcr.jsp

Copyright of Applied Physics Letters is the property of American Institute of Physics and its content may not be copied or emailed to multiple sites or posted to a listserv without the copyright holder's express written permission. However, users may print, download, or email articles for individual use. 